\title{
Analysis of Power Electronics Technology and Reactive Compensation Technology of Power System
}

\author{
Zengxiang Yang ${ }^{1^{*}}$ and Kaipu Yuan ${ }^{2}$ \\ ${ }^{1}$ Wenling Power Supply Company of State Grid, Wenling, Zhejiang 317500, China \\ ${ }^{2}$ Taizhou Power Supply Company of State Grid, Taizhou, Zhejiang 317000, China
}

\begin{abstract}
Rapid development of power electronics technology, successive emerging of new materials and new structural devices, and improvement of computer technology provide vigorous support for actual application of modern control technology. The researches on application of power electronics technology in power system become increasingly extensive and profound, thus enormously supporting and developing reactive compensation technology of power system. This article has discussed application and development of power electronics technology in power system, analyzed reactive power and harmonic wave in power system, briefly introduced the development and research of reactive compensation technology at home and abroad, intensively promoted the development and application of SVC device, and put forward a series of design solutions to lower avoidable energy loss of the whole power system.
\end{abstract}

\author{
KEYWORDS \\ Power system \\ Power electronics technology \\ Reactive compensation \\ Harmonic wave \\ SCR
}

\section{Introduction}

Power electronics technology refers to a modern technology that uses power electronic device to convert and control electric energy. It realizes the final conversion of power frequency electric energy of power grid into electric energy of different natures and different purposes so as to adapt to different demands of the ever changing electric devices. Power electronics technology is an emerging interdisciplinary subject since the $20^{\text {th }}$ century. Power system is an important field for application of power electronics technology. Application of power electronics technology in power system involves several major issues such as improvement of power transmission capacity, bettering of electric energy quality, improvement of operational stability reliability and control flexibility of power grid and reduction of loss. During the construction of power grid system of China, insufficient capacity of reactive compensation and unreasonable and unscientific power grid configuration are two most dif-

Copyright ( 2015 Zengxiang Yang amd Kaipu Yuan

doi: $10.18686 /$ esta.v2i1.3

Received: October 4, 2015; Accepted: November 7, 2015; Published online: December 3

This is an open-access article distributed under the terms of the Creative Commons Attribution Unported License (http://creativecommons.org/ licenses/by-nc/4.0/), which permits unrestricted use, distribution, and reproduction in any medium, provided the original work is properly cited.

${ }^{*}$ Corresponding author: Wenling Power Supply Company of State Grid, Wenling, Zhejiang 317500, China. E-mail: yzx5124@139.com ficult challenges. The issue of insufficient capacity of reactive power is especially serious. The emerging of these problems reflects an unstable status of power grid system, which forces us to continuously develop and research reactive compensation device in power system and realize stable and safe operation of the power system [1].

\section{Harmonic Wave and Reactive Power in Power System} 2.1. Generation of Harmonic Wave and Reactive Power In the current industry and daily life, most electrical loads are resistance-inductance loads. For example, asynchronous motor, fluorescent lamp for daily use, industrial electric arc furnace and transformer are all typical resistanceinductance loads. Resistance-inductance load can work normally only by absorbing reactive power, which is decided by its own nature. Besides, current at AC side of phasecontrolled rectifier, phase-controlled AC power regulatory circuit and cycle converter is also lagged behind voltage. They not only consume abundant reactive power but also generate abundant harmonic current.

In electrical engineering theory, 3 kinds of power are defined for pure sinusoidal AC circuit, namely, active power $\mathrm{P}$, reactive power $\mathrm{Q}$ and apparent power $\mathrm{S}$ as follows:

$$
\begin{gathered}
P=U I \cos \phi \\
Q=U I \sin \phi \\
S=U I
\end{gathered}
$$

Where, $\phi$ refers to phase angle of current being lagged behind voltage. The 3 kinds of power mentioned above sat- 
isfy the following relationship:

$$
S^{2}=P^{2}+Q^{2}
$$

Active power $\mathrm{P}$ indicates average of point of product of instantaneous voltage and instantaneous current (i.e. instantaneous power) within a cycle. The physical significance of active power is AC average power. Apparent power $S$ indicates maximum capacity of electrical equipment and it is the product of effective voltage and effective current. It is used as limit value of power design of electrical equipment in terms of engineering. The rated current is decided by section area of conductor and copper loss, while the rated voltage is decided by insulating property. Reactive power indicates a range of power exchange of circuit or system containing energy storage element. Power exchange in single-phase circuit directly appears between power supply and energy storage equipment (inductance and capacitance). Three-phase circuit is reflected in the back and force flow of load with energy storage character among three phases. The sum of reactive power of three phases at any instant is identically vanishing [2].

As for non-sinusoidal AC circuit, current and voltage can go through Fourier decomposition and can be represented in a series form. Active power and apparent power can still be represented as follows:

$$
\begin{aligned}
& \mathrm{P}=\sum_{n=1}^{\infty} U_{n}^{2} I_{n}^{2} \operatorname{Cos} \psi_{n} \\
& \mathrm{~S}=\mathrm{UI}=\sqrt{\sum_{n=1}^{\infty} U_{n}^{2}} \sqrt{\sum_{n=1}^{\infty} I_{n}^{2}}
\end{aligned}
$$

Where, "Un" and "In" indicate fundamental wave and effective voltage and current of each harmonic wave.

Reactive power of non-sinusoidal circuit containing harmonic wave is relatively complicated and it has not been scientifically and authoritatively defined so far. The reactive power of non-sinusoidal circuit can be defined as follows by referring to pure sinusoidal circuit:

$$
\mathrm{Q}=\sqrt{S^{2}-P^{2}}
$$

Where, reactive power $(\mathrm{Q})$ only reflects energy flow and exchange but does not reflect consumption of energy in load. In the power grid, the wave distortion of voltage is usually very small, while wave distortion of current can be very big. Therefore, it is of realistic significance to study the condition with voltage waveform as sinusoidal wave and current waveform as non-sinusoidal wave without giving consideration to voltage distortion. Therefore, each power can be represented as follows:

$$
\begin{gathered}
\mathrm{P}=\mathrm{U} I_{1} \cos \psi_{1} \quad Q_{f}=\mathrm{U} I_{1} \sin \psi_{1} \\
S^{2}=U^{2} I^{2}=P^{2}+Q_{f}^{2}+U^{2} \sum_{n=2}^{\infty} I_{n}^{2}=P^{2}+Q_{f}^{2}+D^{2} \\
\text { Then, } D^{2}=U^{2} \sum_{n=2}^{\infty} I_{n}^{2}
\end{gathered}
$$

Where, reactive power is decomposed to two parts. "Qf" represents reactive power generated by fundamental current, while $\mathrm{D}$ represents reactive power generated by har- monic current. Therefore, the power factor can be represented as follows:

$$
\lambda=\frac{P}{S}=\frac{I_{1} \cos \psi_{1}}{U I}=\frac{I_{1}}{I} \cos \psi_{1}=v \cos \psi_{1}
$$

Where, " $v=I 1 / I$ " refers to fundamental wave factor or wave distortion factor, and $\cos \phi 1$ is called as fundamental power factor or displacement factor. It is thus clear that power factor of non-sinusoidal current circuit not only depends on phase shift of fundamental current but also is closely related to wave distortion of current, i.e. size of harmonic wave. It is equal to product of fundamental displacement factor and wave distortion factor. Therefore, if harmonic wave is contained in current distortion or circuit, the reactive power will increase and the power factor will decline, so as to result in reduction of availability of electrical capacity of equipment, which is extremely unfavorable for the power distribution network [3].

\subsection{Influence of Reactive Power and Hazards of Har- monic Wave}

The influence of reactive power on power grid is mainly reflected in the following aspects:

(1) Increase of equipment capacity: Increase of reactive power results in increase of current and apparent power so as to increase capacity of engine, transformer, other electrical equipment and conductor.

(2) Increase losses of equipment and line: Increase of reactive power results in increase of total current so as to increase losses of equipment and line.

(3) Increase of voltage drop of line and transformer: Impact reactive power load also leads to violent fluctuation of voltage and seriously reduce power supply quality.

Hazards of harmonic wave to power grid generally include the followings:

(1) Harmonic wave causes harmonic loss to elements in the power grid and therefore reduces efficiency of power generation, power transmission and power utilization equipment. When abundant triple-frequency harmonic flows through neutral line, the line will be overheated and a fire can even be caused.

(2) Harmonic wage influences normal operation of various kinds of electrical equipment. Besides additional loss, harmonic wave also causes mechanical vibration, noise and overvoltage to the motor, makes the transformer locally overheated and leads to aging of insulation and shortening of service life of capacitor and cable to result in damage.

(3) Harmonic wave causes local parallel resonance and series resonance in power grid to enlarge harmonic wave.

(4) Harmonic wave causes wrong action of relay protection and automatic device and leads to inaccuracy of metering of electrical measuring instruments.

(5) Harmonic wave disturbs adjacent communication system. If the condition is not severe, harmonic wave would cause noise to lower communication quality; if the condition is severe, harmonic wave would result in loss of 
information to cause failure of normal operation of communication system [4].

\section{Compensation Principles of Harmonic Suppression and Reactive Power}

In order to solve problems of harmonic pollution and low power factor of power electronic device and other harmonic sources, there are two basic ideas: One is to install compensation device to compensate harmonic wave and reactive power; the other is to improve the power electronic device itself to ensure that it does not generate harmonic wave or consume reactive power, or the power factor is controlled as demanded.

The first idea mentioned above is usually used during current operation process of power system. In other words, reactive power compensation technology should be implemented. Now, we discuss the second idea. The actual function of the second idea is to improve level of whole system and electrical load to further lower internal capacity occupied by electrical equipment as well as maintain degree of voltage of power grid so as to improve the entire power supply quality. The basic principle is very simple and it is to connect capacitive power equipment and inductive electrical load to realize random conversion of power energy between two loads, so as to reduce consumption of reactive power in the whole power grid system.

\section{Current Application Condition of Reactive Power Compensation Device \\ 4.1. Historical Development Progress of Reactive Power Compensation Device}

The initial reactive power compensation devices are only parallel capacitor and synchronously regulated camera. They usually request centralized compensation control in part of system most biased to high voltage. Parallel capacitor is currently the most extensively applied device dedicated to reactive power compensation during development process of power grid. However, due to relative fixation of capacitance, stepless compensation of reactive power of power grid system cannot be realized; besides, since the capacitor has a negative voltage effect, the internal voltage of power grid system drops more quickly; when harmonic wave occurs to the whole power grid system, phenomenon of parallel resonance may take place, so as to enlarge harmonic wave and result in losses.

If viewed from the perspective of further in-depth research, reactive compensation technology in a stationary form is gradually coming into people's sight. Simply speaking, this reactive compensation technology refers to a technology using static switch to implement switching of capacitor or electric reactor and release or converge reactive current so as to improve the power factor of the whole power system in power utilization and finally realize the goal of stability of internal voltage of power grid system.

\subsection{Development and Current Application Condition of SVC Device}

SVC device mainly includes control of SCR towards electric reactor (TCR) and switching of SCR towards capacitor (TSC) as well as combination or the two, or refers to control of SCR towards TCR and mixed matching and use of a series of forms like fixed capacitor or mechanical switching capacitor in the whole power grid system.

\subsubsection{Restriction of SCR towards $T C R$}

The firing angle of SCR is usually continuously regulated and converted from right angle $\left(90^{\circ}\right)$ to flat angle $\left(180^{\circ}\right)$. If the firing angle is increased, the equivalent admittance of TCR will increase accordingly, which can reduce effective compensation of fundamental component of the whole circuit. The amount of TCR circuit develops and changes from fixed value amount to zero. In this case, we can adjust the firing angle to change the actual component of reactive power absorbed by TCR, so as to realize reactive compensation effect of power grid.

\subsubsection{Switching of SCR towards TSC}

The actual principle of TSC circuit is a compensation technology device continuously regulating and absorbing reactive power. In this circuit, the function of two parallelconnected SCR devices is only to combine and involve the capacitor in the power grid or simply disconnect power grid system. Those small inductance devices serially connected are specifically used to inhibit impact current possibly caused. A key principle of switching is that the moment when TSC is switched in must be stipulated as the moment when the system voltage is equal to advance full voltage of capacitor. It voltage of capacitance equipment jumps and changes in a ladder-like way, which can possibly cause current with extremely strong impact to damage SCR and so as to cause a series of unfavorable influences.

\subsubsection{Harmonic Suppression}

Currently, harmonic suppression methods can be classified into two classes:

(1) Compensation method: Reactive LC filter or active power filter

(2) Harmonic source reconstruction method: One is

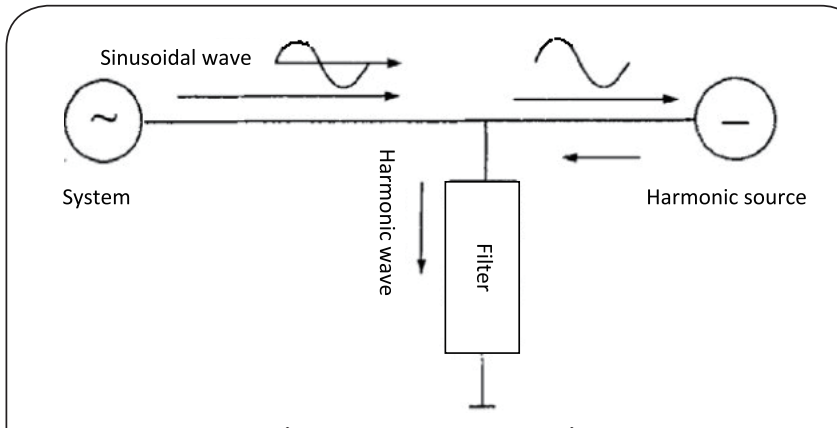

Figure 1. Harmonic source reconstruction. 
to manage to improve phase number of main harmonic source, i.e. rectifying device, in power system; the other is to adopt rectifier with high power factor (Figure 1).

Currently, reactive LC filter is most applied in engineering, for it features simple structure, less investment, high reliability and relatively low operation cost.

The principle of harmonic filtering is essentially provision of a release path for harmonic wave in the circuit (i.e., fundamental wave is reserved to short the harmonic wave so as to make harmonic wave directly flow back through filter without being injected to the system).

\section{Reactive Compensator Based on Variable Reac- tance Static Form}

5.1. Compensation Principle of Static Reactive Compensator Based on Variable Electric Reactor

On the basis of in-depth research on TCR and TSC static reactive power compensators, a member of organization put forward a static reactive power compensation device based on variable reactance in a new subject. It usually comprises stationary capacitor, variable electric reactor and intelligent controller. It features serial connection of SCR and TCR and designing of a variable TCR which mainly comprises variable reactance converter and power converter of power electronics technology. Variable TCR can be divided into primary side and secondary side. The main coil at primary side is usually parallel-connected to load, while coil at secondary coil is usually connected and restricted with power converter of power electronics technology.

The compensator of this technology has the following obvious advantages:

It can utilize variable TCR to continuously adjust reactance value so as to realize stepless objective in power consumption;

This technology isolating high voltage and low voltage can be utilized to lower expectation requirement of system for electronic equipment when control is imposed on low-voltage end, so as to make it convenient to control and save cost of power system. Reactive structure is adopted for device of reactive compensation technology. Compared with active structure, the reactive structure features relatively high operation reliability and relatively low operation cost [5].

\subsection{Topological Structure of Static Reactive Compen- sator Based on Variable TCR}

There are mainly two topological structures of static reactive compensator based on variable TCR at present: Application of SCR or topological structure if IGBT. If the electrical load in power grid system is inductance load, speed of current is lagged behind speed of voltage. Therefore, we have to regulate firing angle of SCR so as to reduce resistance of variable TCR and make it smaller than expected value. At this point, the resistance of reactive power compensator is acceptable. However, if it is parallel-connected to electrical load, its inductive reactance performance will be lowered to increase the power factor. The reactive power factor is relatively small; on the contrary, when the electrical load is capacitive load, speed of current will be ahead of that voltage. Therefore, we have to regulate conduction angle of SCR to increase resistance of variable TCR and make it bigger than expected value. At this point, the resistance of reactive power compensator is inductive. After it is parallel-connected to load, its inductive resistance performance will be increased to make the power factor smaller and the reactive power reactive bigger. Using IGBT and diode as power conversion elements and regulating duty cycle of PWM wave of IGBT trigger pulse, which can change actual impedance of variable TCR so as to realize dynamic form objective of reactive compensation of power grid system [6].

\section{Conclusions}

Rapid development of power electronics technology, successive emerging of new materials and new structural devices, and improvement of computer technology provide vigorous support for actual application of modern control technology. The researches on application of power electronics technology in power system become increasingly extensive and profound, thus enormously supporting and developing reactive compensation technology of power system. During realistic applications, we can use this technology to improve operation rate of whole power distribution network system and also reduce resources and energy consumed in the whole power distribution system to a certain extent, so as to have a favorable energy-saving effect. In the future, power electronics technology will play an increasingly important role in improving reliability and stability of power system.

\section{Conflicts of interest}

These authors have no conflicts of interest to declare.

\section{Authors' contributions}

These authors contributed equally to this work.

\section{References}

1. Niu, Y. N. (2011). Review on the reactive power compensation of power system. Information \& Communications, 01, 55-57.

2. Ge, T. Y. (2010). Engineered application of using SVC for transporting electricity in electric power system. Journal of Ningbo Polytechnic, 02, 23-24.

3. Zhou, B.R., \& Chen, Y. H. (2007). Research on Application of Dynamic Reactive Power Compensators in West-East Electricity Transmission Systems of China Southern Power Grid. Southern Power System Technology, 02, 64-67.

4. Cui, X. Z. (2002), New Application of Power Electronics Technology in Power Development. Energy Technology, 06, 268-270.

5. Wang, Z. A. (1997). Advances of Harmonic Suppression and Reactive Power Compensation Technique for Power Electronic Equipment. Power Electronics, 01, 100-104.

6. Weng, L. M. (2004). Review of Researches on Power Electronics and Harmonics Restraining, Reactive Power Compensation Technique. Power Capacitor, 03, 6-10. 